\title{
Immune Deficiency in Fetal Alcohol Syndrome
}

\author{
SHARRON JOHNSON, RICHARD KNIGHT, DANIEL J. MARMER, AND RUSSELL W. STEELE ${ }^{(18)}$ \\ Section of Immunology and Infectious Diseases, Department of Pediatrics, University of Arkansas for Medical \\ Sciences, Little Rock, Arkansas, and Division of Microbiology and Immunology, Cell Biology Branch HFT 164, \\ National Center for Toxicological Research, Jefferson, Arkansas, USA
}

\section{Summary}

In a review of 13 documented cases of fetal alcohol syndrome (FAS), an increased incidence of life-threatening bacterial infections as well as a propensity to minor infections was observed. Five of 13 patients had had at least one episode of pneumonia, two had meningitis, and one had sepsis. A comprehensive immunologic evaluation of FAS was completed, and results were compared to an age-matched control group of children with intrauterine growth retardation without $F$ AS.

Children with FAS were shown to have decreased $\mathbf{E}$ rosetteforming lymphocytes $(35 \pm 5 \%$ versus $55 \pm 5 \%$ or $1328 \pm 274$ versus $2333 \pm 112$ per $\mathrm{mm}^{3}$ ), low EAC rosette-forming lymphocytes $\left(15 \pm 2 \%\right.$ versus $18 \pm 1 \%$ or $524 \pm 109$ versus $740 \pm 75$ per $\left.\mathrm{mm}^{3}\right)$, and diminished mitogen-induced stimulation responses to mitogens: $31616 \pm 5337$ versus $58076 \pm 4455 \mathrm{cpm}$ for phytohemagglutinin, $17582 \pm 5436$ versus $35018 \pm 5346$ for pokeweed mitogen, and $32460 \pm 7044$ versus $54996 \pm 5531$ for concanavalin $A, P<$ 0.05. Nine patients had dysgammaglobulinemia. FAS subjects also had a marked eosinophilia (624 \pm 154 versus $\left.72 \pm 27 \mathrm{~mm}^{3}\right)$. Other parameters of immune function including absolute lymphocyte and neutrophil counts, total hemolytic complement, delayed cutaneous hypersensitivity, and nitroblue tetrazolium dye reduction assays, were not different from control children. Impairment of immunity may explain an increased susceptibility to infection in FAS.

\section{Speculation}

Defects in host defense and propensity to infection are attributable to intrauterine exposure to high levels of alcohol. Such abnormalities are not related to the degree of postnatal growth retardation nor to degree of malnutrition and do not correct with increasing age. Subsequent disease processes associated with defects in immunity such as malignancy or autoimmunity could result from this exposure to alcohol during fetal development.

There is increasing awareness of a distinct dysmorphic phenotype presently termed the fetal alcohol syndrome (FAS). Since the initial description by Lemoire et al. (10) in France and later by Jones et al. (9) in this country, animal studies $(3,12,17)$ and additional clinical documentation have confirmed ethanol as the most common teratogen causing mental retardation in the United States and Europe (4). The incidence of FAS is estimated to be 1 to $2 / 1000$ with incomplete expression in an additional 3 to $5 / 1000$ live births.

Previous investigations have focused primarily on the morphological and neurological features of the syndrome and excellent reviews have been published $(4,14)$. Recently, in a large genetics referral clinic, we appreciated a high frequency of acquired overt infectious diseases among children with FAS. This observation has not been noted in published reports. We therefore selected patients of various ages who satisfied current criteria for FAS, reviewed clinical histories, and examined many aspects of immune function in an effort to define deficiencies that might account for a propensity to infection.

\section{MATERIALS AND METHODS}

\section{STUDY GROUP AND CONTROL POPULATIONS}

Thirteen children and infants with FAS were evaluated. Ages ranged from 12 months to $101 / 2$ years. All demonstrated a majority of those clinical features described with FAS (Table 1). In addition, the family history for all study patients included excessive alcohol consumption by the mother. None of the study patients was malnourished at the time of examination. Thirteen age- and sex-matched normal children, seen for routine care as part of another clinical investigation, made up one control group. Most FAS patients in this study were small for gestational age (SGA). Because assays of cellular immunity have in some studies been shown to be depressed in children with intrauterine growth retardation, an additional matched control group of thirteen SGA patients without FAS was included; the cause of growth retardation in all of these study cases was placental insufficiency; two resulted from maternal toxemia, one from maternal hypertension, two from hereditary SGA, one from maternal excessive smoking, and seven were unknown. All of these youngsters were above the 10th percentile for height and weight. For both control groups, ages of individually matched children were within $10 \%$ of FAS study cases.

\section{IMMUNOLOGIC ASSAYS}

Delayed cutaneous hypersensitivity was examined with two intradermally administered skin test antigens, Candida albicans (1:100 and 1:10; Hollister-Stier) and tetanus toxoid (1:1, MerrillNational Laboratories). Reactions greater than $5 \mathrm{~mm}$ induration were considered positive.

The microassay methods selected for this study allowed all in vitro tests to be completed for patients and controls with approximately $5 \mathrm{ml}$ of peripheral venous blood and included basic measurement of cellular and humoral immunity as well as assays for phagocytic function.

Total leukocyte and differential counts were completed by routine methodology. Quantitative immunoglobulin levels were assessed by laser nephelometry and total hemolytic complement $\left(\mathrm{CH}_{100}\right)$ measurement by radial diffusion using Quantiplates (Kallestad Lab). Spontaneous and endotoxin-stimulated nitroblue tetrazolium (NBT) dye reduction was performed using the semiquantitative slide method (6).

Lymphocytes were separated on a Hypaque-Ficoll gradient. In vitro lymphocyte-stimulation responses to the mitogens, phytohemagglutinin, pokeweed, and concanavalin A were determined by micromethods previously described (16). Briefly, $0.1 \mathrm{ml}$ of lymphocyte suspensions $\left(2 \times 10^{5}\right.$ lymphocytes) was added to an equal volume of various concentrations of mitogens in sterile flat-bottom microtest plates. A harvesting apparatus previously described and technically refined for our use was used for separation of the stimulated lymphocytes on glass-fiber filters for washing of these cells and for recovery of radioactive $\left[{ }^{3} \mathrm{H}\right]$ thymidine uptake (15). Results are reported as average counts per minute (cpm) of triplicate samples from those concentrations of mitogens demon- 
Table 1. Features of FAS in 13 infants and children

\begin{tabular}{lc}
\hline \multicolumn{1}{c}{ Feature } & No. affected \\
\hline Growth deficiency & \\
Prenatal & 8 \\
Postnatal & 9 \\
& \\
Central nervous system dysfunction & \\
Neurological-microcephaly & 6 \\
Intellectual-mental retardation & 6 \\
Behavioral-hyperactivity or irritability & 6 \\
Facial characteristics & \\
Short palpebral fissures & \\
Hypoplastic philtrum & 9 \\
Thinned upper vermillion & 3 \\
Retrognathia & 3 \\
Maxillary hypoplasia & 2 \\
& 2 \\
Associated features & \\
Epicanthal folds & \\
Cardiac anomalies & 4 \\
Ear anomalies & 7 \\
Skeletal anomalies & 3 \\
Hirsuitism in infancy & 4 \\
High arched palate & 3 \\
Cleft palate & 3 \\
\hline
\end{tabular}

Table 2. Infectious diseases experienced by 13 infants and children with FAS

\begin{tabular}{lc}
\hline \multicolumn{1}{c}{ Infectious process } & No. affected \\
\hline Sepsis & 1 \\
Meningitis & 2 \\
Soft tissue infection ${ }^{1}$ & 3 \\
Pneumonia $^{\text {Other infection }}{ }^{2}$ & 5 \\
Recurrent otitis media & 6 \\
Frequent upper respiratory infection & 9 \\
\hline${ }^{1}$ Cellulitis, abscesses, or impetigo. & 11 \\
${ }^{2}$ Urinary tract infection, gastroenteritis, or monilial diaper rash.
\end{tabular}

strating maximum stimulation responses. Lymphocytes incubated with medium alone showed some variation from one individual to another with cpm ranging between 500 and 1200 . Because small differences in these control values can greatly influence a stimulation index, gross cpm are reported in this study.

Percentages and absolute numbers of circulating lymphocytes with receptors for $\mathrm{C}^{3} \mathrm{~b}$ (erythrocyte-antibody complement, EAC rosettes) and for sheep red blood cells ( $E$ rosettes) were determined according to the methods of Mendes et al. (11). The former method measures predominantly $B$ cells although monocytes and $\mathrm{K}$ cells may bear receptors for the $\mathrm{C}_{3}$ component of complement.

\section{RESULTS}

\section{CLINICAL DATA}

Infectious diseases documented in the FAS study group are summarized in Table 2. Most had had systemic involvement or infection of visceral organs. Two infants had bacterial meningitis, and one of these manifest generalized sepsis. Of the five children with a history of lobar pneumonia, four had two separate episodes, and the fifth patient had three documented recurrences. Repeated otitis media and three or more episodes per year of upper respiratory infection accompanied by fever were seen in the majority of subjects. There was no apparent correlation between clinical infections and severity of immune deficiency.

\section{IMMUNOLOGIC PARAMETERS}

Assays used to examine cell-mediated immunity included: (1) absolute lymphocyte counts; (2) E rosettes; (3) mitogen-induced stimulation, and (4) skin reactivity to recall antigens. Of these parameters, all were depressed (Table 3) except results for skindelayed hypersensitivity. Only two of the 13 children with FAS failed to react to the skin antigens as compared to only one of the 13 SGA controls and none of the appropriate for gestational age (AGA) controls. Decreased numbers of total circulating lymphocytes could be attributable to intrauterine growth retardation alone because results were not significantly different from those for SGA controls $(P>0.05)$. Although mitogen-induced stimulation responses were reduced in FAS as compared to both AGA and SGA children, there was no significant difference between the two control groups.

Table 3. Immune parameters for 13 infants and children with FAS compared to 13 age- and sex-matched appropriate for gestational age and 13 SGA controls

\begin{tabular}{|c|c|c|c|c|c|}
\hline Immune parameters & $\begin{array}{l}\text { AGA } \\
\text { controls }\end{array}$ & $P$ & $\begin{array}{c}\text { FAS } \\
\text { study population }\end{array}$ & $\boldsymbol{P}$ & $\begin{array}{c}\text { SGA } \\
\text { controls }\end{array}$ \\
\hline Absolute neutrophils $/ \mathrm{mm}^{3}$ & $3272 \pm 909$ & NS & $3606 \pm 1017$ & NS & $3380 \pm 725$ \\
\hline \multicolumn{6}{|l|}{$\mathrm{E}$ rosettes } \\
\hline$\%$ & $63.7 \pm 6.8$ & $<0.001$ & $35.5 \pm 5.4$ & $<0.001$ & $55.0 \pm 5.1$ \\
\hline \multicolumn{6}{|l|}{ EAC rosettes } \\
\hline$\%$ & $19.6 \pm 2.3$ & $<0.001$ & $14.7 \pm 1.9$ & $<0.05$ & $18.1 \pm 1.1$ \\
\hline Absolute & $1094 \pm 128$ & $<0.001$ & $524 \pm 109$ & $<0.05$ & $740 \pm 75$ \\
\hline \multicolumn{6}{|l|}{ Mitogen stimulation (cpm) } \\
\hline Phytohemagglutinin & $53924 \pm 6797$ & $<0.005$ & $31616 \pm 5337$ & $<0.001$ & $58076 \pm 4455$ \\
\hline Stimulated & $68.3 \pm 14.2$ & NS & $65.8 \pm 15.0$ & NS & $59.4 \pm 17.1$ \\
\hline
\end{tabular}

\footnotetext{
${ }^{1}$ Mean \pm S.D.
} 
Table 4. Abnormalities ${ }^{1}$ of immunoglobulins in 13 cases of FAS

\begin{tabular}{lc}
\hline \multicolumn{1}{c}{ Dysgammaglobulinemia } & No. affected \\
\hline Normal & 4 \\
Low IgG & 2 \\
Low IgA & 3 \\
Low IgM & 1 \\
Low IgG and IgA & 2 \\
Low IgG, IgA and IgM & 1 \\
\hline
\end{tabular}

\footnotetext{
${ }^{1}$ More than one standard deviation below age- and sex-matched control
} values.

Screening for humoral immunity included: (1) EAC rosettes; (2) quantitative immunoglobulin determination; and (3) total hemolytic complement. The absolute number of B (EAC) lymphocytes was significantly lower in the FAS subjects compared to AGA children and SGA controls (Table 3). Various abnormalities in the concentrations of $\operatorname{IgG}, \operatorname{IgA}$, and $\operatorname{IgM}$ were seen in nine of 13 study cases with FAS, and individual results are presented in Table 4. A decreased response is defined as one standard deviation below control values. Three subjects had decreased $\operatorname{IgA}$, two had decreased IgG, and one had decreased IgM. Two additional subjects had both decreased $\operatorname{IgG}$ and $\operatorname{IgA}$. One had decreased $\operatorname{IgG}, \operatorname{Ig} A$, and IgM. No control study cases had abnormal quantitative immunoglobulin levels. Total hemolytic complement was within the normal range for all subjects.

Testing for neutrophil function included total neutrophil counts and NBT assays. Absolute neutrophil counts were not significantly different between study populations nor was spontaneous and endotoxin stimulated NBT dye reduction.

Absolute number of eosinophils was significantly increased in FAS population compared to both AGA $(P<0.001)$ and SGA $(P<0.001)$ populations.

\section{DISCUSSION}

The present study has suggested that children with FAS have a greatly increased susceptibility to both life-threatening and also minor infectious diseases. Two episodes of bacterial meningitis among 13 study cases is, by itself, a striking observation. In addition, five others required hospitalization for lobar consolidated pneumonia and three for gastroenteritis.

Recurrent otitis media occurred in approximately $70 \%$ of FAS children as compared to an incidence of 10 to $17 \%$ in the general pediatric population as reported previously (13). Frequent upper respiratory infection documented in 11 of 13 study patients appears excessive although the significance of minor infections is, of course, difficult to assess. No patients had infections with opportunistic organisms such as Pneumocystis carinii nor unusual manifestations of childhood viral infection such as chickenpox.

Another possible factor predisposing FAS children to infection is the relatively high frequency of congenital heart disease. Although seven FAS cases had congenital defects, only one would be considered of clinical importance. Moreover, as a group, the children with heart defects did not demonstrate clinical or laboratory parameters different from those without such findings.

Numerous immune deficiencies were documented in our FAS population which may account for the clinical observation of

Table 5. An individual comparison of immunologic and clinical data in 13 cases of FAS

\begin{tabular}{|c|c|c|c|}
\hline Patient & $\begin{array}{l}\text { Age } \\
(\mathrm{yr})\end{array}$ & Abnormal immunologic studies & Clinical findings \\
\hline T. B. & 1 & Decreased absolute neutrophil count and $\mathrm{E}$ rosettes & Meningitis, recurrent otitis media, frequent $\mathrm{URI}^{1}$ \\
\hline V. H. & $1 L_{2}$ & $\begin{array}{l}\text { Decreased absolute neutrophil count, E rosettes, and EAC } \\
\text { rosettes }\end{array}$ & Persistent monilia diaper rash \\
\hline M. C. & 2 & Decreased $\mathrm{IgA}, \mathrm{E}$ rosettes, and EAC rosettes & Pneumonia $\times 2$, recurrent otitis media, frequent URI \\
\hline E. C. & 3 & $\begin{array}{l}\text { Decreased absolute neutrophil and lymphocyte counts, } \\
\operatorname{IgG} \text {, and } \operatorname{IgA}, \mathrm{E} \text { rosettes and EAC rosettes }\end{array}$ & Pneumonia $\times 2$, recurrent otitis media, frequent URI \\
\hline J. C. & 3 & $\begin{array}{l}\text { Decreased absolute lymphocyte count, } \operatorname{IgG} \text { and } \operatorname{IgA}, \mathrm{E} \\
\text { rosettes and EAC rosettes }\end{array}$ & $\begin{array}{l}\text { Pneumonia } \times 1 \text {, urinary tract infection, recurrent otitis } \\
\text { media, frequent URI }\end{array}$ \\
\hline S. S. & 4 & Decreased absolute lymphocyte count, and IgA & Recurrent otitis media, and frequent URI \\
\hline D. $S$. & 5 & $\begin{array}{l}\text { Decreased absolute neutrophil count, IgM, E rosettes, EAC } \\
\text { rosettes, negative skin test responses }\end{array}$ & Meningitis, sepsis, frequent URI \\
\hline J. S. & $5 \frac{1}{2}$ & $\begin{array}{l}\text { Decreased absolute lymphocyte count IgG, IgA, and IgM, } \\
\text { E rosettes and EAC rosettes }\end{array}$ & $\begin{array}{l}\text { Recurrent otitis media, frequent URI, chronic gastroenter- } \\
\text { itis }\end{array}$ \\
\hline C. $\mathrm{S}$. & 7 & $\begin{array}{l}\text { Decreased absolute neutrophil and lymphocyte counts and } \\
\text { IgG }\end{array}$ & $\begin{array}{l}\text { Recurrent otitis media, recurrent impetigo, persistent "dia- } \\
\text { per rash" }\end{array}$ \\
\hline M. N. & 10 & $\begin{array}{l}\text { Decreased absolute lymphocyte count, IgA, E rosettes, } \\
\text { EAC rosettes, negative skin tests }\end{array}$ & Recurrent otitis media, frequent URI \\
\hline
\end{tabular}

\footnotetext{
${ }^{1}$ URI, upper respiratory infection.
} 
increased infectious diseases (Table 5). Defects in cellular immunity are known to be associated with viral and fungal processes such as upper respiratory infections and monilial diaper rash whereas decreased humoral immune defense would predispose the host to sepsis, meningitis, pneumonia, and otitis media. IgA deficiency is also an important factor in the host's susceptibility to both upper and low respiratory infection and gastroenteritis; six of 13 FAS children were IgA deficient. Although absolute neutrophil counts and resting and stimulated NBT determinations were normal, more extensive examination of neutrophil function is needed to more fully evaluate this aspect of immunity. Many of the observed clinical infectious processes, particularly soft tissue infection, suggest possible abnormalities of phagocytic mechanisms.

The FAS children in our series had absolute lymphocyte counts comparable to SGA controls with both groups significantly lower than the AGA controls. This suggests that intrauterine growth retardation alone accounted for lymphopenia. However, other parameters were significantly lower in the FAS as compared to the SGA group. These included $\mathrm{E}$ rosettes, mitogen stimulation, EAC rosettes, and quantitative immunoglobulin determinations. Thus, the major immunotoxic effect appears to be intrauterine exposure to ethanol.

As different from previous studies (7), no differences were seen in the SGA as compared to the AGA control groups for mitogeninduced stimulation and delayed cutaneous hypersensitivity. However, present results are consistent with data demonstrating decreased absolute lymphocyte counts and $\mathrm{E}$ rosettes but a normal percentage of EAC rosetting lymphocytes in SGA patients (7). Variations in skin test antigen preparations and methodology may account for the former discrepancies.

Other investigators have also suggested that intrauterine malnutrition from a variety of causes may result in persistent impairment of immunologic competence. Sequential studies for up to one year have demonstrated hypogammaglobulinemia and cellmediated immunity (2). The older ages of the present FAS patients suggest that postnatal effects from alcohol may be longer lasting than other recognizable causes of malnutrition.

Perhaps of greatest clinical importance is recognition of hypogammaglobulinemia $\mathrm{G}$ in FAS because this deficiency can be readily corrected with administration of immune serum globulin. More than one-third of FAS patients in the present series had IgG determinations below one standard deviation for normal controls. However, none of the FAS patients had levels below $250 \mathrm{mg} / \mathrm{d}$, that range where gammaglobulin replacement might be considered (5). One patient (E.C., Table 5) had levels below three standard variations at $284 \mathrm{mgm} / \mathrm{dl}$ and a history including two episodes of lobar pneumonia.

Barrett et al. (1) have recently reported two patients with classic DiGeorge Syndrome whose mothers had histories of excessive alcohol consumption. It is intriguing that the facial and clinical features of FAS (Table 1) are strikingly similar to the character- istics of DiGeorge syndrome. Furthermore, the present data suggest an overlap of immunologic abnormalities for these two clinical entities. No abnormalities of calcium metabolism were noted in FAS patients.

Protein-calorie malnutrition in children has been shown to markedly decrease mechanisms of cell-mediated immunity, but this effect is reversible with proper dietary therapy (8). In contrast, the diffuse defects noted in FAS did not appear to be related to the degree of postnatal growth retardation nor degree of malnutrition and did not appear to correct with increasing age. Longer follow-up is needed to determine whether deficiencies persist into adulthood. If this is the case, other conditions associated with defects in immunity such as neoplastic and autoimmune processes would be anticipated.

\section{REFERENCES AND NOTES}

1. Barrett, D. J., Ammann, A. J., Wara, D. W., Cowan, M. J., Fisher, T. J., and Steihm, E. R.: Clinical and immunologic spectrum of the DiGeorge Syndrome (in preparation).

2. Chandra, R. K.: Immunocompetence in low-birth-weight infants after intrauterine malnutrition. Lancet, 2: 1393 (1974).

3. Chernoff, G. F.: The fetal alcohol syndrome in mice: an animal model. Teratology, 15: 223 (1977).

4. Clarren, S. K., and Smith, D. W.: The fetal alcohol syndrome. N. Engl. J. Med., 298: 1063 (1978).

5. Committee on Infectious Diseases: Primary immunodeficiencies and use of immune serum globulin (human). In: Report of the Committee on Infectious Diseases pp. 15-18 (American Academy of Pediatrics, Evanston, IL, 1977)

6. Feigin, R. D., Shackelford, P. G., Choi, S. C., Flake, K. K., Franklin, F. A., Jr., and Eisenberg, C. S.: Nitroblue tetrazolium dye test as an aid in the differential diagnosis of febrile disorders. J. Pediatr., 78: 230 (1971).

7. Ferguson, A. C.: Prolonged impairment of cellular immunity in children with intrauterine growth retardation. J. Pediatr. 93: 52 (1978).

8. Ferguson, A. C., Lawlor, G. J., Neumann, C. G., Oh, W., and Stiehm, E. R.: Decreased rosette-forming lymphocytes in malnutrition and intrauterine growth retardation. J. Pediatr., 85: 717 (1974).

9. Jones, K. L., Smith, D. W., Ulleland, C. N., and Streissguth, A. P.: Pattern of malformation in offspring of chronic alcoholic mothers. Lancet, $1: 1267$ (1973).

10. Lemoire, P., Harousseau, H., Borteyre, J. P., and Menuet, J. C.: Les enfants de parents alcoliques: anomalies observées. Quest. Med., 24: 476 (1968).

11. Mendes, N. F., Tolnai, M. E. A., and Silveria, N. P. A.: Technical aspects of the rosette tests used to detect human complement receptor (B) and sheep erythrocyte-binding (T) lymphocytes. J. Immunol., 111: 860 (1973).

12. Sander, S.: The influence of ethyl alcohol on the developing chick embryo. II, Rev. Roum. Embryol., 5: 167 (1968).

13. Shurin, P. A., Pelton, S. I., and Finkelstein, B. A.: Tympanometry in the diagnosis of middle ear effusion. N. Engl. J. Med., 296: 412 (1977)

14. Smith, D. W.: The fetal alcohol syndrome. Hosp. Pract., 14: 121 (1979).

15. Steele, R. W., Hensen, S. A., Vincent, M. M., Fuccillo, D. A., and Bellanti, J. A. $A^{51} \mathrm{Cr}$ microassay technique for cell-mediated immunity to viruses. J. Immunol., 110: 1502 (1973).

16. Steele, R. W., Limas, C., Thurman, G. B., Bauer, H., and Bellanti, J. A.: Familial thymic aplasia: attempted reconstitution with fetal thymus in a Millipore diffusion chamber. N. Engl. J. Med., 287: 787 (1972).

17. Tze, W. J. and Lee, M.: Adverse effects of maternal alcohol consumption on pregnancy and foetal growth in rats. Nature (Lond.), 257: 479 (1975).

18. Requests for reprints should be addressed to: Russell W. Steele, M.D., Department of Pediatrics, University of Arkansas for Medical Sciences, 4301 West Markham Street, Little Rock, Arkansas 72205 (USA).

19. Received for publication March 25, 1980

20. Accepted for publication September 15, 1980. 